\title{
The age of reason for FDG PET image-derived indices
}

\author{
Dimitris Visvikis • Mathieu Hatt • Florent Tixier • \\ Catherine Cheze Le Rest
}

Received: 15 August 2012 / Accepted: 17 August 2012 /Published online: 12 September 2012

(C) Springer-Verlag 2012

The clinical use of PET imaging using ${ }^{18} \mathrm{~F}$-FDG is currently predominantly focused on diagnosis in the field of oncology. In this context, image analysis is largely based on visual interpretation and the use of simple image-derived indices, such as the maximum standardized uptake value $\left(\mathrm{SUV}_{\max }\right)$, which corresponds to the voxel with the maximum activity concentration in the tumour scaled by the administered activity, patient weight and blood glucose concentration. On the other hand, during the last few years there has been increasing interest in the use of ${ }^{18} \mathrm{~F}$-FDG PET imaging for the prediction and monitoring of therapy response. In this context the $\mathrm{SUV}_{\max }$ has been also predominantly used, where differences between a pretreatment and posttreatment scan have been shown to closely correlate with clinical response to treatment in a number of different cancer models $[1,2]$.

The clear advantage of such a simplistic image-derived index is the ease of use, which has significantly contributed to its widespread application in clinical practice. $\mathrm{SUV}_{\max }$ is also in principle less dependent on partial volume effects (PVE) resulting from the limited spatial resolution of PET imaging [3]. On the other hand, there are a number of issues associated with its use, such as lack of robustness in terms of image noise, as well as in terms of the reconstruction algorithm and the associated corrections used during the reconstruction process. Clearly the impact of these issues can be minimized by standardization of the injection, acquisition, and image reconstruction and analysis protocols. In

D. Visvikis $(\bowtie) \cdot$ M. Hatt $\cdot$ F. Tixier

LaTIM, INSERM UMR1101, CHRU MORVAN,

5 avenue Foch,

29609 Brest, France

e-mail: dimitris@univ-brest.fr

C. Cheze Le Rest

Department of Nuclear Medicine, CHU Milétrie,

Poitiers, France addition, the use of peak SUV ( $\left.\mathrm{SUV}_{\text {peak }}\right)$, calculated by averaging the voxel values inside a small region of interest centred on the tumour maximum activity concentration voxel, can reduce the sensitivity of $\mathrm{SUV}_{\max }$ to noise, although results may be sensitive to the actual definition of the ROI used to compute $\mathrm{SUV}_{\text {peak }}$ [4]. However and most importantly, $\mathrm{SUV}_{\max }$ represents only very limited information in considering radiotracer accumulation and not considering information on the associated tumour uptake distribution or on the overall tumour functional volume. This is true even in the context of static acquisitions associated with routine whole-body ${ }^{18} \mathrm{~F}$-FDG PET imaging, where dynamic acquisitions, which offer the possibility, through kinetic modelling, of deriving quantitative glucose metabolic rates on a voxel-by-voxel basis, are not considered.

Remaining with the context of static PET imaging, overall tumour activity accumulation can be alternatively characterized using the mean SUV ( $\left.\mathrm{SUV}_{\text {mean }}\right) . \mathrm{SUV}_{\text {mean }}$ corresponds to the mean activity concentration in a delineated three-dimensional (3D) functional tumour volume. The most significant issues associated with the accuracy of $\mathrm{SUV}_{\text {mean }}$ determination include the precision and robustness in the delineation of the tumour functional volume used $[5,6]$, and the important influence of PVE which is closely related to the overall tumour size. The impact of PVE becomes more significant in studies of response to therapy where the metabolically active tumour volume (MATV) may change during treatment, consequently introducing a variable influence of PVE on the calculated difference in $\mathrm{SUV}_{\text {mean }}$ between the baseline and the posttreatment image. All these dependence issues, combined with the lack of robust tools for tumour volume segmentation and PVE correction in PET oncology imaging, have previously limited the widespread use of $\mathrm{SUV}_{\text {mean }}$.

A combination of the MATV and the $\mathrm{SUV}_{\text {mean }}$, defined as the total lesion glycolysis (TLG) or total glycolytic volume originally proposed by Larson et al. [7], allows in principle a 
more comprehensive functional tumour characterization. The advantage of such an index is that it includes the average level of uptake in the tumour in addition to the spatial extent of the tumour uptake distribution, which facilitates discrimination between lesions of similar size but different uptake levels and vice versa. The potential clinical impact of using such a parameter, reflecting overall tumour metabolic information rather than a single or few voxel measures based on $\mathrm{SUV}_{\max }$ or $\mathrm{SUV}_{\text {peak }}$, respectively, has recently been demonstrated. TLG levels derived from a baseline PET image were able to predict, in contrast to $\mathrm{SUV}_{\text {max }}$, response to therapy in different cancer models, including mesothelioma [8], lymphoma [9] and oesophageal carcinoma [10].

However, despite their potential value, image-derived indices such as 3D MATV and TLG have found only limited use up to date, including in the context of recent recommendations for response to therapy assessment using ${ }^{18} \mathrm{~F}-\mathrm{FDG}$ PET imaging [11]. This can be explained mostly by the limited accuracy, robustness and reproducibility of the clinically available tumour delineation tools. The shortcomings of standard threshold-based approaches for MATV, which are currently the only widely available approaches in clinical practice, have been highlighted in the literature $[6,12]$. They include a strong dependence on overall tumour volume and contrast. On the other hand, manual MATV delineation is a time-consuming process prone to high inter- and intraoperator variability [13]. This renders manual tumour delineation a nonviable tool for allowing the introduction of MATV as an image-derived index in routine clinical practice. Different automatic segmentation approaches have recently been proposed, motivated by the interest in using ${ }^{18} \mathrm{~F}$ FDG PET for MATV delineation in the field of radiotherapy treatment planning [14]. Some of these approaches have shown high robustness and reproducibility with PET images of variable quality (depending on factors such as scanner sensitivity and image reconstruction parameters) and tumour activity distribution characteristics, allowing physiological reproducibility limits similar to those with $\operatorname{SUV}_{\text {max }}[13,15]$. In addition, the use of these approaches has shown promising results for the use of MATV measures in the prediction and prognosis of response to therapy based on ${ }^{18} \mathrm{~F}-\mathrm{FDG}$ PET imaging [6, 10]. However, being able to accurately determine a $3 \mathrm{D}$ functional tumour volume does not obviate the need for PVE correction to ensure the calculation of an accurate tumour $\mathrm{SUV}_{\text {mean. }}$ This extra step clearly further complicates the process of accurately determining TLG values.

For all these reasons, a novel parameter, called standardized added metabolic (SAM) activity recently proposed by Mertens and colleagues [16] in the European Journal of Nuclear Medicine and Molecular Imaging, may be of particular interest. The attractiveness of this parameter is its potential independence from an accurate MATV determination as well as PVE. Its derivation is based on considering the product of the concentration of activity and corresponding volume in two manually defined concentric volumes of interest (VOIs). These VOIs are placed around the tumour at a sufficient distance from the MATV border to avoid PVE. The concentration of activity in the difference VOI is used to determine the background activity which is subtracted from the $\mathrm{SUV}_{\text {mean }}$ derived using the VOI closer to the tumour. Although this parameter contains information related to both MATV and $\mathrm{SUV}_{\text {mean }}$, it does not provide precise 3D tumour volume measurements and as such does not require an accurate MATV segmentation algorithm or manual tumour delineation. At the same time the mean tumour activity concentration calculated in the context of SAM is in principle not influenced by PVE. In this same study, it was also shown that SAM is less influenced by noise than $\mathrm{SUV}_{\max }$. Finally, a potential dependence on the scanner calibration and activity injection parameters can be excluded using a "normalized SAM" version by dividing the calculated tumour SAM value by the mean background SAM, assuming that the background and the tumour are equally influenced by the different acquisition factors.

A preliminary clinical evaluation of this new PET imagederived parameter was performed as part of the same study [16] considering 19 patients with colorectal carcinoma undergoing baseline and treatment follow-up PET scans (after three to six chemotherapy cycles). In this patient population, consisting of only responders and nonresponders, no statistically significant differences were found between $S_{U V} V_{\max }$ and SAM for assessing response to therapy based on PET imaging. This was the case considering either the baseline PET image values or differences between the baseline and the follow-up scans. More studies are now necessary considering different cancer models and larger and more variable patient populations in order to demonstrate the value of $\mathrm{SAM}$, not only compared to $\mathrm{SUV}_{\max }$ but also in comparison to other ${ }^{18} \mathrm{~F}$-FDG PET image-derived parameters such as $3 \mathrm{D}$ MATV and TLG values in terms of both prediction and assessment of response to therapy.

One can imagine going even further in exploiting tumour activity distribution features extracted from static PET images. Different levels of heterogeneity in tumour uptake distribution could be assessed, which could eventually correlate with the underlying physiological processes governing tumour function. Since some of these physiological processes are potential mediators or targets of different therapeutic regimens, such image-derived indices could also be proposed in the context of therapy response prediction and prognosis. Some recent studies have shown the interest in such indices for predicting response to therapy in head and neck [17], cervical [17] and oesophageal cancer [18]. 
Finally, it may be appropriate to combine multiple PET image-derived indices and even multimodality and/or multitracer image-derived parameters for a more complete tumour characterization. Complex correlations will have to be considered given the complexity of underlying tumour biology. Molecular profiling of biological specimens today allows the expression levels of multiple genes and proteins to be revealed. Obtaining biological specimens requires invasive procedures and may depend on the location of the biopsy within a tumour. Will it ever be possible to use multimodality imaging, combining different image-derived indices identified as "signatures" for the expression of particular genes. as an alternative technique for noninvasive molecular profiling of individual cancers? This is still a dream, but based on a few existing studies, we may consider the realization of this idea in the future as a target [19]. Only the future will tell...

In conclusion, we have available today a number of algorithms and approaches that allow the automatic or semiautomatic extraction of accurate quantitative parameters from the tumour activity distribution in static ${ }^{18} \mathrm{~F}-\mathrm{FDG}$ PET images. We have in this sense reached the age of reason in PET image-derived parameters. There is now an urgent need for these tools to become widely available in order to allow the clinical research community to demonstrate their potential for therapy assessment, therapy response prediction and patient survival in different cancer models. A first step in this process involves standardization, robustness and reproducibility studies for all these "novel" PET imagederived indices in comparison with current "state of the art" parameters in clinical use such as $\mathrm{SUV}_{\max }$.

\section{References}

1. Weber WA. Assessing tumor response to therapy. J Nucl Med. 2009;50 Suppl 1:1S-10S.

2. Michalski MH, Chen X. Molecular imaging in cancer treatment. Eur J Nucl Med Mol Imaging. 2011;38(2):358-77.

3. Tylski P, Stute S, Grotus N, Doyeux K, Hapdey S, Gardin I, et al. Comparative assessment of methods for estimating tumor volume and standardized uptake value in 18F-FDG PET. J Nucl Med. 2010;51:268-76.

4. Vanderhoek M, Perlman SB, Jeraj R. Impact of the definition of peak standardized uptake value on quantification of treatment response. J Nucl Med. 2012;53(1):4-11.

5. Krak NC, Boellaard R, Hoekstra OS, Twisk JW, Hoekstra CJ, Lammertsma AA. Effects of ROI definition and reconstruction method on quantitative outcome and applicability in a response monitoring trial. Eur J Nucl Med Mol Imaging. 2005;32(3):294-301.
6. Hatt M, Visvikis D, Albarghach NM, Tixier F, Pradier O, Cheze-le Rest C. Prognostic value of 18F-FDG PET imagebased parameters in esophageal cancer and impact of tumor delineation methodology. Eur $\mathrm{J}$ Nucl Med Mol Imaging. 2011;38(7):1191-202.

7. Larson SM, Erdi Y, Akhurst T, Mazumdar M, Macapinlac HA, Finn RD, et al. Tumor treatment response based on visual and quantitative changes in global tumor glycolysis using PET-FDG imaging: the visual response score and the change in total lesion glycolysis. Clin Positron Imaging. 1999;2:159-71.

8. Lee HY, Hyun SH, Lee KS, Kim BT, Kim J, Shim YM, et al. Volume-based parameter of (18)F-FDG PET/CT in malignant pleural mesothelioma: prediction of therapeutic response and prognostic implications. Ann Surg Oncol. 2010;17(10):2787-94.

9. Cazaentre T, Morschhauser F, Vermandel M, Betrouni N, Prangère T, Steinling M, et al. Pre-therapy 18F-FDG PET quantitative parameters help in predicting the response to radioimmunotherapy in non-Hodgkin lymphoma. Eur J Nucl Med Mol Imaging. 2010;37(3):494-504.

10. Hatt M, Visvikis D, Pradier O, Cheze-le Rest C. Baseline 18FFDG PET image-derived parameters for therapy response prediction in oesophageal cancer. Eur J Nucl Med Mol Imaging. 2011;38:1595606.

11. Wahl RL, Jacene H, Kasamon Y, Lodge MA. From RECIST to PERCIST: evolving considerations for PET response criteria in solid tumors. J Nucl Med. 2009;50 Suppl 1:122S-50S.

12. Nestle U, Kremp S, Schaefer-Schuler A, Sebastian-Welsch C, Hellwig D, Rübe C, et al. Comparison of different methods for delineation of 18F-FDG PET-positive tissue for target volume definition in radiotherapy of patients with non-small cell lung cancer. J Nucl Med. 2005;46(8):1342-8.

13. Hatt M, Cheze Le Rest C, Aboagye EO, Kenny LM, Rosso L, Turkheimer FE, et al. Reproducibility of 18 F-FDG and 18F-FLT PET tumour volume measurements. J Nucl Med. 2010;51 (9):1368-76.

14. Zaidi H, El Naqa I. PET-guided delineation of radiation therapy treatment volumes: a survey of image segmentation techniques. Eur J Nucl Med Mol Imaging. 2010;37(11):2165-87.

15. Hatt M, Cheze Le Rest C, Albarghach N, Pradier O, Visvikis D. PET functional volume delineation: a robustness and repeatability study. Eur J Nucl Med Mol Imaging. 2011;38(4):663-72.

16. Mertens J, Dobbeleir A, Ham H, D’Asseler Y, Goethals I, Van de Wiele C. Standardized added metabolic activity (SAM): a partial volume independent marker of total lesion glycolysis in liver metastases. Eur J Nucl Med Mol Imaging. 2012;39(9):1441-8. doi:10.1007/s00259-012-2166-0.

17. El Naqa I, Grigsby PW, Aptea A, Kidd E, Donnelly E, Khullar D, et al. Exploring feature-based approaches in PET images for predicting cancer treatment outcomes. Pattern Recognit. 2009;42:1162-71.

18. Tixier F, Le Rest CC, Hatt M, Albarghach N, Pradier O, Metges JP, et al. Intratumor heterogeneity characterized by textural features on baseline 18F-FDG PET images predicts response to concomitant radiochemotherapy in esophageal cancer. J Nucl Med. 2011;52 (3):369-78.

19. Segal E, Sirlin CB, Ooi C, Adler AS, Gollub J, Chen X, et al. Decoding global gene expression programs in liver cancer by noninvasive imaging. Nat Biotechnol. 2007;25:675-80. 